\title{
Field Theory of Spin-Singlet Quantum Hall States
}

\author{
Kazusumi Ino \\ Nomura Research Institute,Hongo 2-2-9,Bunkyo-ku, Tokyo,113-0033, Japan
}

\begin{abstract}
We formulate a field theory for a class of spin-singlet quantum Hall states which have been proposed for the quantized Hall plateaus observed at the second lowest Landau level (the Haldane-Rezayi state and its variants). A new essential ingredient is a class of super Chern-Simons field. We show that the known properties of the states are consistently described by it. We also give a $2+1$ dimensional hierarchical construction. Implications of the proposal are discussed and a new physical picture of composite particles emerges.

PACS.73.40 Hm, 11.30 Pb, 11.25 Hf
\end{abstract}

Introduction Recently, new phenomena in the high Landau levels of the two-dimensional electron gas attract much attention [1]. Among them is the quantized Hall plateau found at $\nu=5 / 2$, where $\nu$ is the filling of Landau levels [2, 3] and its denominator is even. This is a unique exception to the odd-denominator rule-the fractional quantized Hall plateaus are always found around filling fractions with odd-denominators.

Haldane and Rezayi [4] proposed an ansatz wave function for the $\nu=5 / 2$ state which is spin-singlet. The tilted field experiments show a collapse of the gap in accordance with the proposal of Haldane and Rezayi [5]. Although it has some attractive features as a candidate for the $\nu=5 / 2$ plateau, it was argued [6] that the hollowcore model in which the ansatz wave function gives the exact ground state is not a good approximation to the system with Coulomb interaction at the second lowest Landau levels $(\mathrm{N}=1 \mathrm{LL})[\mathrm{G}]$.

Numerical studies [8] of the Coulomb model show that the ground state at $\nu=5 / 2$ may be the spin-polarized state proposed by Moore and Read [9]. This direction attracts some attention 10 12. However, the discussions pose problems in some important respects.

First of all, as this state is spin-polarized, a new explanation for the results of tilted field experiments is required. Until now, some suggestions such as the effect of thickness of samples have been made. Although the role of spin at $\mathrm{N}=1 \mathrm{LL}$ is largely unknown, it has been pointed out that, in specimens to realize fractional quantum Hall effect (FQHE), the effective Zeeman energy can be so small that the spin degrees of freedom are not frozen out [13]. Another unsatisfactory feature is in its failure of implementing the idea of hierarchy 14]. In view of Jain's successful idea and the prominent role of $\nu=1 / 2,1 / 4, \cdots$ composite Fermi liquid [15] in FQHE at the lowest Landau level $(\mathrm{N}=0 \mathrm{LL})$, it has been proposed that FQHE at $\mathrm{N}=1$ LL may be also dictated by a hierarchy of very different kind [16].

The state also has a difficulty in $2+1$ dimensional field theoretical interpretation. There have been some proposals of $2+1$ dimensional field theory all based on $S U(2)$ Chern-Simons gauge theory at level 2 11 (except the one proposed in Ref. [12]). A problem in these proposals is that they are valid only at special unrealistic filling fraction, or require an elimination of superfluous degrees of freedom, of which a physical meaning is unclear. This stems from the fact that the pairing in the state is given by two-dimensional $c=1 / 2$ Majorana fermion for which no direct $2+1$ dimensional interpretation is known.

For a class of spin-singlet quantum Hall states, namely permanent quantum Hall states (variants of the HaldaneRezayi state [17]) are identified as hierarchical states formed on the Haldane-Rezayi state [16]. These states inherit most features of the HR state. Indeed, the recent experiments at the plateaus around $\nu=5 / 2$ $(\nu=7 / 3,8 / 3 \ldots)$ suggest a close relation between these plateaus [3].

In this paper, we propose a $2+1$ dimensional field theory of the Haldane-Rezayi state and its variants (permanent quantum Hall states). A new essential ingredient is a class of super Chern-Simons field. We show that the known properties of the states are consistently described by it. We also give a $2+1$ dimensional hierarchical construction. Implications of the proposal are discussed and new physical picture of composite particle at $\mathrm{N}=1 \mathrm{LL}$ emerges.

Permanent quantum Hall state For the FQHE at $\mathrm{N}=0 \mathrm{LL}$, wave functions were given by conformal blocks of 2 dimensional conformal field theory in the bulk $\left(\mathrm{CFT}_{2}\right)$ while edge excitations were described by $1+1$ dimensional theory on the edge $\left(\mathrm{CFT}_{1+1}\right)$. There $\mathrm{CFT}_{2}$ and $\mathrm{CFT}_{1+1}$ were equivalent and the global symmetry of CFT was generated by the gauge symmetry of $2+1$ dimensional CS theory. These are all based on the relation between the CS theory and CFT [18]. We expect that this scheme is at work also for $\mathrm{N}=1 \mathrm{LL}$, but with one extension. In the $\mathrm{HR}$ or permanent $\mathrm{QH}$ states, $\mathrm{CFT}_{2}$ and $\mathrm{CFT}_{1+1}$ are slightly different although their space of states coincides and the partition functions are equal with an inclusion of non-trivial flux [19 21]. This flux is only accounted for by the twist of the boundary condition in $\mathrm{CFT}_{1+1}$. Thus the field content of the $2+1$ dimensional theory will respect the symmetry of $\mathrm{CFT}_{2}$. We shall use this as a guiding principle for constructing a proper $2+1$ dimensional field theory. Especially the boundary action deduced from the $2+1$ dimensional theory should be the one for $\mathrm{CFT}_{2}$.

Let us recall $\mathrm{CFT}_{2}$ of the $\nu=1 / q(q=p+1, p$ :even 
integer) permanent $\mathrm{QH}$ state. As observed in [9], the pairing part of the permanent state can be written as a conformal block of $c=-1$ bosonic ghosts $\beta-\gamma$ [26]. In terms of these fields and a chiral boson $\varphi$, the electrons in the permanent state are represented by $\beta e^{i \sqrt{q} \varphi}, \quad \gamma e^{i \sqrt{q} \varphi}$. There is a bosonization scheme for the $\beta-\gamma$ system [26 16$]$ in which $\beta$ and $\gamma$ are bosonized into $c=-2$ symplectic fermion system and a chiral boson $\phi$ with negative signature as follows:

$$
\beta=\partial \theta^{\uparrow} e^{\phi}, \quad \gamma=\partial \theta^{\downarrow} e^{-\phi} .
$$

$\phi$ and $\varphi$ may be generated by U(1) CS fields in $2+1$ dimensional theory. For the $c=-2$ part, there is a ParisiSoulas supersymmetry, which indicates an emergence of a fermionic gauge symmetry in $2+1$ dimensions.

These considerations lead us to consider the generators of gauge symmetry $P^{\mu}, Q, \widetilde{Q}(\mu=0,1)$ where $P^{\mu}$ is for $\mathrm{U}(1)$ symmetry, $Q$ and $\widetilde{Q}$ are for possible supersymmetry. We assume that the U(1) gauge symmetries commute $\left[P^{\mu}, P^{\nu}\right]=0$. To construct a proper commutation relations involving $Q$ and $\widetilde{Q}$ one must take into account an important observation in Ref. 16] that the formation of filled LL for composite fermions should affect the pairing. A simple way to implement it is to consider $Q$ and $\widetilde{Q}$ forming a representation of $P^{\mu}$. However, the effect of filled LL on pairing is noninvertible in the sense that the converse effect that filled LL affects unpaired composite fermions to form a pair do not occur. But the converse effect is inevitable when $Q$ and $\widetilde{Q}$ form a representation. To avoid it, we may take $\widetilde{Q}$ as a fermionic central extension of an ordinary supersymmetry algebra as follows:

$$
\left[P^{\mu}, P^{\nu}\right]=0, \quad\{Q, Q\}=2 \gamma_{\mu} P^{\mu}, \quad\left[P^{\mu}, Q\right]=2 i \gamma^{\mu} \widetilde{Q},
$$

where we set $\gamma^{0}=\gamma^{1}=-\gamma_{0}=\gamma_{1}=1$. Other commutatators are all set to zero. These commutation relations satisfy the Jacobi identity and form a graded Lie algebra. We may define an invariant metric by the trace

$$
\operatorname{Tr}\left(P^{\mu} P^{\nu}\right)=-\frac{1}{2} \eta^{\mu \nu}, \operatorname{Tr}(\widetilde{Q} Q)=\frac{i}{2} .
$$

In (2), supercharges appear unsymmetrically. To treat them in a symmetric way, we may take a basis in spin by $Q^{\uparrow}=\frac{1}{\sqrt{2}}(Q+\widetilde{Q}), \quad Q^{\downarrow}=\frac{1}{\sqrt{2}}(Q-\widetilde{Q})$. Similar enlarged gauge symmetry has been known in supergravity 222 and also studied in the relation between CS theory and superstring theories 23].

The one-form gauge field has the expansion

$$
\mathcal{A}=i A^{\mu} P_{\mu}+\psi Q-\widetilde{\psi} \widetilde{Q},
$$

where $A^{\mu}$ is $\mathrm{U}(1)$ gauge field, $\psi$ and $\widetilde{\psi}$ are real fermionic gauge fields. We consider the CS action for $\mathcal{A}$ ( with $k=1$ ),

$$
\begin{aligned}
S & =\frac{k}{4 \pi} \int \operatorname{Tr}\left(\mathcal{A} d \mathcal{A}+\frac{2}{3} \mathcal{A}^{3}\right) \\
& =\frac{k}{4 \pi} \int \frac{1}{2} A^{\mu} \wedge d A_{\mu}+i \widetilde{\psi} \wedge d \psi+i \psi \wedge \gamma^{\mu} \psi \wedge A_{\mu} .
\end{aligned}
$$

The coupling to a source is achieved by the addition of a term $\mathcal{J} \wedge \mathcal{A}$. The field equation is $d \mathcal{A}+[\mathcal{A}, \mathcal{A}]=\mathcal{J}$, which is in components

$$
\begin{aligned}
& d A^{\mu}+i \psi \wedge \gamma^{\mu} \psi=J^{\mu}, \quad d\left(\psi_{\uparrow}+\psi_{\downarrow}\right)=J_{Q}, \\
& d\left(\psi_{\uparrow}-\psi_{\downarrow}\right)-2\left(\psi_{\uparrow}+\psi_{\downarrow}\right) \gamma^{\mu} \wedge A_{\mu}=J_{\widetilde{Q}} .
\end{aligned}
$$

The third equation tells that when two sources with fermions with opposite spins get closer, they will be deflected by the bosonic gauge fields. This is a kind of dynamics supposed to happen in the permanent states.

The action has the gauge symmetry $\delta \mathcal{A}=d \Lambda+[\mathcal{A}, \Lambda]$ on a closed space. However on a space with boundary such as cylinder, it is necessary to add a boundary action. We will now show that it actually coincides with the action of $\mathrm{CFT}_{2}$ of the permanent state. To derive it, we note that the field equation with no source is solved by $\mathcal{A}_{\alpha}=\mathcal{U}^{-1} \partial_{\alpha} \mathcal{U}, \quad \mathcal{U}=\exp (X), \quad X=i \varphi^{\mu} P_{\mu}+i \theta Q-i \widetilde{\theta} \widetilde{Q}$. In components, $A_{\alpha}^{\mu}=\partial_{\alpha} \varphi^{\mu}-i \theta \gamma^{\mu} \partial_{\alpha} \theta, \psi_{\alpha}=\partial_{\alpha} \theta, \widetilde{\psi}_{\alpha}=$ $\partial_{\alpha} \widetilde{\theta}+\gamma^{\mu} \theta \partial_{\alpha} \varphi_{\mu}-\gamma^{\mu} \partial_{\alpha} \theta \varphi_{\mu}$. By using the solution, we get the boundary action for the cylinder $\left(g_{+-}=g_{-+}=-1\right)$

$$
\begin{aligned}
S_{\mathrm{B}} & =-\int\left\{A_{+}^{\mu} A_{-\mu}+i \psi^{\alpha} \widetilde{\psi}_{\alpha}\right\} d^{2} \sigma+i \int \epsilon^{\alpha \beta \gamma} \psi_{\alpha} \gamma_{\mu} \psi_{\beta} A_{\gamma}^{\mu} d^{3} \sigma \\
& =-\int\left\{\left(\partial_{+} \varphi^{\mu}-i \theta \gamma^{\mu} \partial_{+} \theta\right)\left(\partial_{-} \varphi_{\mu}-i \theta \gamma_{\mu} \partial_{-} \theta\right)\right. \\
& \left.+i \partial^{\alpha} \theta\left[\partial_{\alpha} \widetilde{\theta}+\gamma^{\mu} \theta \partial_{\alpha} \varphi_{\mu}-\gamma^{\mu} \partial_{\alpha} \theta \varphi_{\mu}\right]-i \epsilon^{\alpha \beta} \partial_{\alpha} \varphi^{\mu} \theta \gamma_{\mu} \partial_{\beta} \theta\right\} d^{2} \sigma .
\end{aligned}
$$

Calculation shows that some terms including four fermi terms cancel. Then we have

$S_{\mathrm{B}}=-\int\left\{\partial_{+} \varphi^{\mu} \partial_{-} \varphi_{\mu}+i \partial^{\alpha} \theta \partial_{\alpha} \widetilde{\theta}-i \epsilon^{\alpha \beta} \partial_{\alpha} \varphi^{\mu} \theta \gamma_{\mu} \partial_{\beta} \theta\right\} d^{2} \sigma$.

In (8), we notice that a field redefinition of $\widetilde{\theta}$ by $\widetilde{\theta}+\theta F$ generates a term similar to the last one. By taking $F$ satisfying $\partial_{\alpha} F=\gamma^{\mu} \epsilon_{\alpha \beta} \partial^{\beta} \phi_{\mu}$, we end up with

$$
S_{\mathrm{B}}=-\int\left\{\partial_{+} \varphi^{\mu} \partial_{-} \varphi_{\mu}+i \partial^{\alpha} \theta \partial_{\alpha} \widetilde{\theta}\right\} d^{2} \sigma .
$$

This is the action for $\mathrm{CFT}_{2}$ of the $\nu=1$ permanent state. For the $\nu=1 / q$ state, we add additional CS term $\frac{p}{2} A^{1} \wedge d A^{1}$ to the Lagrangian. This is because $A^{1}$ gives rise to the Laughlin-Jastrow factor for filled LL in the ground state wave function which, for the Laughlin state, comes from the CS field for flux attachment.

The field redefinition to get the action (9) spoils the explicit relation between the local supersymmetry and the global supersymmetry $Q_{\text {global }}^{a}, P_{\text {global }}^{0}, P_{\text {global }}^{1}$. But the correspondence can be given by characterizing representations by the charges under two U(1) symmetries and the fermions.

Thus we have shown that the CS theory based on the algebra (2) leads to the correct $\mathrm{CFT}_{2}$ of the permanent $\mathrm{QH}$ state. This implies that it is a proper field theory for the state. For $\mathrm{CFT}_{1+1}$, we should take the corresponding 
unitary theory which has the same partition function as the $\mathrm{CFT}_{2}[16]$.

(Quasi-) Particles from the Wilson lines In Ref. [24], it was realized that earlier concept of flux attachment 25] is promoted to vortex attachment. This is natural from the CS field theoretic point of view since composite (quasi-)particles can now be expressed by the Wilson lines. This is readily extended to our case.

The Wilson line in our system is

$$
W=\operatorname{Tr}_{\mathcal{R}} P \exp \left(\int_{C} \mathcal{A}\right)
$$

where $P$ indicates the path-ordered product, $C$ is a contour and $\operatorname{Tr}_{\mathcal{R}}$ is the trace for a representation of $\mathcal{R}$ of the source. Wave functions are obtained as an expectation value in the presence of $W$ with the position braket at a time slice. For $N$ Wilson lines, their current is a sum of delta functions at their contours $\mathcal{J}=\sum_{i=1}^{N} j^{s} T_{s} \delta\left(C_{i}\right)$ where $T_{s}$ represents the generators of the gauge group. We identify the species of Wilson lines by their representation under $Q_{\text {global }}^{a}, P_{\text {global }}^{0}$ and $P_{\text {global }}^{1}$, denoting them by the fermions and the charges as $\left(\psi_{s}^{a}, q^{0}, q^{1}\right)$. Here $s$ represents the holonomy for the fermion.

First of all, we consider $\left(\psi_{\mathrm{R}}^{\uparrow}, 1, q\right),\left(\psi_{\mathrm{R}}^{\downarrow},-1, q\right)$ where $R$ represents the trivial holonomy. From the relation between $\mathrm{CS}$ theory and the $\mathrm{CFT}_{2}$, these Wilson lines correspond to $\partial \theta^{\uparrow} e^{\phi} e^{i \sqrt{q} \varphi}, \partial \theta^{\downarrow} e^{-\phi} e^{i \sqrt{q} \varphi}$ i.e. composite fermions in the system. The ground state of the permanent QH state for $2 N$ electrons is obtained as a collection of $N\left(\psi_{\mathrm{R}}^{\uparrow}, 1, q\right)$ and $N\left(\psi_{\mathrm{R}}^{\downarrow},-1, q\right)$ (with uniform background field for $A^{1}$ ).

Let us next consider quasiparticles. For the paired state, the elementary quasiparticle will appear with flux halved. It is a configuration of $A^{1}$ with a holonomy $e^{i \pi}$. Also such quasiparticle generates a non-trivial phase shift for $A^{0}$ or $\psi^{a}$. As shown in Ref. [16], the consistency of the edge theory admits the existence of such a configuration only for $\psi^{a}$. For $\psi^{a}$, the holonomy in the configuration is $e^{i \pi}$. We denote this species as $\psi_{\mathrm{NS}}$. Thus the elementary quasiparticle and quasihole are characterized as the species $\left(\psi_{N S}^{a}, 0,-1\right),\left(\psi_{N S}^{a}, 0,1\right)$.

It is possible to characterize the paired condensate by gauge symmetry breaking. For FQHE at $\mathrm{N}=0 \mathrm{LL}, \mathrm{U}(1)$ gauge symmetry is spontaneously broken by the condensation of composite boson 25]. In our case, two species of composite fermions condensate. The super gauge symmetry is divided into two parts one of which vanishes in the configuration of one species of composite fermion. A collection of one species breaks half of the super gauge symmetry. When both species are present, both parts of the super gauge symmetry are broken.

We also note that half of the supersymmetry which doesn't vanish in the configuration of one species of composite fermion produce a fermionic zero mode in the configuration. The configuration with a fermionic zero mode gives the remaining species of quasiparticle in the system. It corresponds to the logarithmic field in the $\mathrm{CFT}_{2}$.
Hierarchy The construction we have considered can be extended to hierarchical scheme. As in FQHE at $\mathrm{N}=0$ LL, we introduce $m$ kinds of flux described by $m$ U(1) CS gauge field $A^{l}(l=1, \cdots, m)$. The couplings between $\varphi_{i}$ are specified by an integer matrix $K$ 27,28]. The Jain's hierarchy is obtained by taking $K$ as $K_{l n}= \pm \delta_{l n}+p C_{l n}$ where $p$ is an even integer and $C_{l n}=1$ for $l, n=1, \cdots, m$. The filling fraction is given by $\nu=m /(m p \pm 1)$. As shown in 28], this system of U(1) CS fields is equivalent to the system of $\mathrm{SU}(\mathrm{m}) \mathrm{CS}$ field at level 1 and U(1) CS field at level $1 / \nu$. As above, we extend this $\mathrm{U}(1)$ symmetry to (2) by introducing fermionic CS fields $\psi, \widetilde{\psi}$ and bosonic field $A^{0}$. The composite fermions are expressed by the Wilson lines of $\left(\psi^{\uparrow}, \mathbf{1}, 1,1 / \nu\right),\left(\psi^{\downarrow}, \mathbf{1},-1,1 / \nu\right)$ where $\mathbf{1}$ stands for the fundamental representation of $\mathrm{SU}(\mathrm{m})$. The quasiparticles under the presence of the collection of composite fermions reproduce the ones given in 16$]$.

The Haldane-Rezayi state Let us turn to the $\nu=$ $1 / p$ HR state. The permanent state is formed by integer QHE (IQHE) on the HR state. Then, for the HR state, one expect that $Q$ and $P$ may be decoupled since no LL for composite fermion is formed there. This may be simulated by scaling $P$ to zero. However it also requires the vanishment of $\widetilde{Q}$ through the algebra (2), thus does not lead to a sensible construction.

Actually alternative viewpoint is possible: the $\mathrm{HR}$ state is a permanent QH state with IQHE turned off. This results in an algebraic relation of wave functions. It is known that the determinant of the HR wave function and the permanent factor of the permanent state wave function have a simple relation [4]

$\operatorname{det}\left(\frac{1}{\left(z_{i}^{\uparrow}-z_{j}^{\downarrow}\right)^{2}}\right)=\operatorname{per}\left(\frac{1}{z_{i}^{\uparrow}-z_{j}^{\downarrow}}\right) \operatorname{det}\left(\frac{1}{z_{i}^{\uparrow}-z_{j}^{\downarrow}}\right)$.

Here $\operatorname{det}\left(\frac{1}{z_{i}^{\uparrow}-z_{j}^{\downarrow}}\right)$ is interpreted as the factor to "turn off' IQHE. It is the same as the pairing part of the 331 state [13, and thus arises from the U(1) CS theory at level 1 . Its generator actually coincides with $P^{1}$ in the algebra (2). For the permanent QH state, we also took $P^{1}$ to implement the even number of flux for composite fermion. For the HR state, we do not do so not to change the level of the U(1) CS gauge field. Thus we take the algebra (2) for the gauge symmetry of the pairing part only, and take another U(1) CS field $A^{2}$ at level $p$ for the flux of composite fermion. Composite fermions are given by the Wilson lines of $\left(\psi_{R}^{\uparrow}, 1,1, p\right)$ and $\left(\psi_{R}^{\downarrow},-1,-1, p\right)$

At first sight, it does not correspond to the known $c=$ $-2+1 \mathrm{CFT}_{2}$ of the $\mathrm{HR}$ state since it contains additional two bosons for pairing part and gives $c=0+1$. Evidences that it actually does describe the HR state are in order.

First the chiral operator product algebra (OPA) generated by the CS theory coincides with the known OPA of the HR state. Since the OPA determines the correlation between quasiparticles, their statistics and topological order, it implies that the proposed theory is describing the 
HR state. Also the cylinder partition function generated by the OPA using the fermionic character formula agrees with the one for $\mathrm{CFT}_{2}$ except for the contribution from the central charge.

Second evidence is concerned with the central charge. In Ref. [18], it was shown that the so-called 'flaming" dependence of the phase of the partition function of a CS theory determines the central charge. For convenience, we take the large $k$ limit and consider one-loop calculation. It involves the determinant of the fermions. The proposed model has a nice feature that the fermions couples to the bosonic CS fields off-diagonally, thus the absolute value of the fermion determinant and the determinant from boson cancel in the pairing part. Thus the flaming dependence of the pairing part comes only from the phase of the fermion determinant and gives $c=-2$. This value of the central charge is also necessary to achieve the modular invariance of the cylinder partition function by the twisting mechanism of Ref. [21], since, otherwise, each part of the partition function wouldn't transform linearly under the modular transformations. This indicates the necessity of a correction to the naive counting of the central charge in the extended version of the CS-CFT correspondence. For the permanent state, half of the phase dependence is shown to be canceled by the boson determinant, and thus the flaming dependence tells that it has $c=-1+1$. These calculations are expected to be one-loop exact by virtue of supersymmetry. The flaming dependences also indicate the consistency of our proposal.

In this theory, spin degrees of freedom interact with the U(1) CS field. It is expected as in Ref. [16] for the permanent QH states, but is unexpected for the HR state. The algebra (2) may be intrinsic to spin-singlet pairing.

We remark that the characterization of the paired condensate via spontaneous breaking of the super gauge symmetry is valid also for the HR state. Accordingly the concept of composite particle may be modified. At $\nu=1 / 2$, composite fermion is formed by electron and vortex while, at $\nu=5 / 2$, composite fermion may be formed by electron and supersymmetric vortex (Fig.11).

Discussions We add a matter to our theory by the Wilson lines, which may be suitable for an application to knot theory. It is also possible to couple a secondquantized matter by the supermultiplet containing complex boson while respecting the local symmetry. We plan to address a precise formulation in a subsequent paper. Such a formulation enables a quantitative study of the dynamical mechanism of pair formation while qualitative behavior can be deduced within our approach as we now describe.

Perturbative treatment of quasiparticle at the $\nu=1 / 2$ state [15] is justified by the renormalization group flow of a scaling onto the Fermi surface [29] with the proviso of renormalization of the effective mass. Based on this, in Ref. 30, it was shown that the U(1) CS field is highly pair breaking. For the Coulomb interaction, one must resort to a softening of the interaction by the thickness of real specimen for pairing to occur.

Now let us apply the same method to our case. In our case, strong fluctuation of the U(1) CS gauge field for a spin-singlet pair is suppressed by the contribution from the fermionic gauge field for spin. Thus the pair breaking effect is largely tamed for the spin-singlet pair. This reduction of pair breaking effect of composite fermions suggests a similar mechanism at the microscopic level. Actually an example of such mechanism is already known : the HC model 伯. There the pairing is induced by the reduction of zero-th order pseudopotential between electrons. Our observation implies that, if a HC-like model is really valid at $\mathrm{N}=1 \mathrm{LL}$, it should involve spin as its essential cause. Indeed, tilted field experiments have been suggesting that the spin degrees of freedom may be important at $\mathrm{N}=1 \mathrm{LL}$.

Finally it may be interesting to apply our construction to similar planer systems such as given in Ref. 31,32. We plan to address this issue [33].

Acknowledgement The author is grateful to Mahito Kohmoto for useful discussions and to Takashi Nakano at RCNP of Osaka University for correspondence.

[1] See J. P. Eisenstein et al., cond-mat/0003405 for a review.

[2] R. G. Clark et al, Surf. Sci. 170 (1986) 141 ; R. L. Willet et al., Phys. Rev. Lett. 59 (1987) 1776.

[3] W. Pan et al. Phys. Rev. Lett. 838591.

[4] F. D. M. Haldane and E. H. Rezayi, Phys. Rev. Lett. 60 (1988) 956; ibid 1886.

[5] J.P. Eisenstein et al., Phys. Rev. Lett. 61 (1988) 997; J.P. Eisenstein et al. Surf. Sci.229 (1990) 31.

[6] A. H. MacDonald, D.Yoshioka and S. M. Girvin, Phys. Rev. B39 (1989) 1932.

[7] The Landau levels are classified by N. The correspponding noninteracting electronic energy levels are $(N+$ $1 / 2) \hbar \omega_{c}$ where $\omega_{c}$ is the cyclotron frequency $e B / m_{e} c$. $B$ is the magnetic field and $m_{e}$ is the effective mass of electrons. The lowest Landau level is $N=0 \mathrm{LL}$ and the second lowest Landau level is $N=1 \mathrm{LL}$. Since electrons have spin degree of freedom, each Landau level has $\nu=2$ if it is totally filled by electrons with up and down spins. (The Zeeman splitting is neglected here.)

[8] M. Greiter, X.-G.Wen and F. Wilczek, Phys. Rev. Lett. 66 (1991)3205 ; Nucl. Phys. B374 (1992) 567 ; R. H. Morf, Phys. Rev. Lett. 80 (1998) 1505.

[9] G. Moore and N. Read, Nucl. Phys. B360 (1991) 362.

[10] N. Read and D. Green, Phys. Rev. B61 (2000) 10267.

[11] E. Fradkin et al.,Nucl. Phys. B516, (1998)704 ; E. Fradkin et al., Nucl. Phys. . B546, (1999)711 ; D. C. Cabra et al., cond-mat/9905192.

[12] X.-G. Wen, cond-mat/9811111.

[13] B. I. Halperin, Helv. Phys. Acta, 56 (1983) 75. 
[14] F. D. M. Haldane, Phys. Rev. Lett. 51 (1983) 609 ; B. I. Halperin,ibid. 52 (1983) 1583 ; J. K. Jain, ibid. 63 (1989) 199 ; Phys. Rev. B40 (1989) 8079.

[15] B.I. Halperin, P.A. Lee, and N. Read, Phys. Rev. B47, 7312 (1993).

[16] K. Ino, Phys. Rev. Lett. 83 (1999) 8596.

[17] D. Yoshioka, A.H. MacDonald and S.M.Girvin, Phys. Rev. B38 (1989) 3636.

[18] E. Witten, Commun.Math.Phys 121 (1989) 351 ; G. Moore and N. Seiberg, Phys. Lett. B220 (1989) 422.

[19] M. Milovanović and N. Read, Phys. Rev. B53 (1996) 13559.

[20] V. Gurarie, M. Flohr and C. Nayak, Nucl. Phys. B498 (1998) 513 .

[21] K. Ino, Phys. Rev. Lett. 82 (1999) 4902.

[22] R. D'Auria and P. Fré, Nucl. Phys. B201 (1982) 101.

[23] M. B. Green, Phys. Lett. B223 (1989) 157.

[24] N. Read, Semicond. Sci. Technol. 9 (1994) 1859; R. Shankar and G. Murthy, Phys. Rev. Lett. 79 (1997) 4437.

[25] S. C. Zhang, T. H. Hansson and S. Kivelson, Phys. Rev. Lett. 62 (1989) 82.

[26] D. Friedan, E. Martinec and S. Shenker, Nucl. Phys. B271 (1986) 93

[27] N. Read, Phys. Rev. Lett. 65 (1990) 1502.

[28] J. Fröhlich and A. Zee, Nucl. Phys. B364 (1991) 517; X. -G. Wen and A. Zee, Phys. Rev. B46 (1992) 2290.

[29] C. Nayak and F. Wilczek, Nucl. Phys. B430 (1994) 534.

[30] N. E. Bonesteel, Phys. Rev. Lett. 82 (1999) 984.

[31] L. Balents, M.P.A. Fisher and C. Nayak, Int. J. Mod. Phys. B12 (1998) 1033 ; Phys. Rev. B 60 (1999) 1654 ; Phys. Rev. B 61 (2000) 6307.

[32] T. Senthil and M. P. A. Fisher, cond-mat/9910224 to appear in Phys. Rev. B.

[33] K. Ino, work in progress.
$\mathbf{N}=\mathbf{0} \mathbf{L L}$

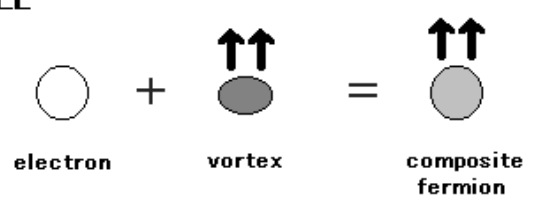

\section{$\mathbf{N}=1 \mathbf{L L}$}

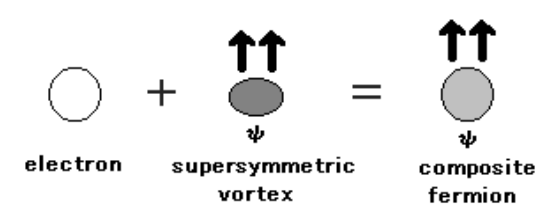

FIG. 1. At N=0 LL, composite fermion is formed by electron and vortex. At $\mathrm{N}=1 \mathrm{LL}$, composite fermion may be formed by electron and supersymmetric vortex. 